\title{
Overexpression of ribonuclease inhibitor induces autophagy in human colorectal cancer cells via the Akt/mTOR/ULK1 pathway
}

\author{
YING TANG ${ }^{1}$, FENG REN $^{2}$, XI CONG $^{2}$, YING KONG $^{2}$, YUXIANG TIAN $^{2}$, YUEFEI XU $^{2}$ and JIANHUI FAN ${ }^{2}$ \\ ${ }^{1}$ Department of Pathology, The Second Affiliated Hospital of Dalian Medical University, Dalian, Liaoning 116023; \\ ${ }^{2}$ Department of Biochemistry and Molecular Biology, Dalian Medical University, Dalian, Liaoning 116044, P.R. China
}

Received June 19, 2018; Accepted March 6, 2019

DOI: $10.3892 / \mathrm{mmr} .2019 .10030$

\begin{abstract}
Ribonuclease inhibitor (RI), also termed angiogenin inhibitor, acts as the inhibitor of ribonucleolytic activity of RNase A and angiogenin. The expression of RI has been investigated in melanoma and bladder cancer cells. However, the precise role of RI in tumorigenesis, in addition to RI-induced autophagy, remains poorly understood. In the present study, it was demonstrated that RI positively regulated autophagy in human colorectal cancer (CRC) cells as indicated by an increase in light chain 3 (LC3)-II levels. Furthermore, RI regulated cell survival in HT29 cells. In addition, autophagy-associated proteins, including beclin-1 and autophagy-related protein 13 , were increased in response to RI-induced autophagy, and the protein kinase B (Akt)/mechanistic target of rapamycin (mTOR)/Unc-51 like autophagy activating kinase (ULK1) pathway may be involved in the activation of autophagy induced by RI overexpression. Taken together, the evidence of the present study indicated that the overexpression of RI induced ATG-dependent autophagy in $\mathrm{CRC}$ cells via the Akt/mTOR/ULK1 pathway, suggesting that the upregulation of RI activity may constitute a novel approach for the treatment of human colorectal carcinoma.
\end{abstract}

\section{Introduction}

Colorectal cancer (CRC) ranks as the third most common cancer in the United States (1) and the fifth greatest cause of mortality in China in 2015 (2). Despite marked improvements in therapeutic strategies (3), the prognosis of patients with CRC remains poor. Thus, novel molecular targets that improve patient outcomes or serve as standard prognostic factors are warranted.

Correspondence to: Dr Jianhui Fan, Department of Biochemistry and Molecular Biology, Dalian Medical University, 9 South Lvshun Road Western Section, Dalian, Liaoning 116044, P.R. China E-mail: fanjh@dlmedu.edu.cn

Key words: ribonuclease inhibitor, colorectal cancer, autophagy, signal pathway
The human ribonuclease inhibitor (RI) is a $50-\mathrm{kDa}$ horseshoe-shaped protein that acts as the inhibitor of the ribonucleolytic activity of RNaseA and angiogenin (ANG) (4-7). The expression of RI has been investigated in melanoma and bladder cancer cells $(8,9)$. Based on its ability to inhibit angiogenesis, RI has been considered as a tumor suppressor. Previous studies have indicated that exogenous RI prolongs the survival time of B16 melanoma tumor-bearing mice (10), and RI expression has been demonstrated to be significantly reduced in human breast cancer tissues (11). However, to date, the mechanism underlying RI expression and tumorigenesis remains unclear. It was previously demonstrated that the overexpression of RI suppresses proliferation and metastasis in human CRC cells via the phosphoinositide 3-kinase (PI3K)/protein kinase B (Akt) pathway (12). A recent study has also suggested that autophagy is activated when Akt is inhibited (13).

Autophagy, a cellular process that degrades cytoplasmic components via the lysosomal machinery, is morphologically characterized by the formation of light chain 3 (LC3)-II autophagic vacuoles $(14,15)$. In tumorigenesis, autophagy acts as a double-edged sword that may either promote cell survival or induce cell death (16). However, previous studies have also indicated that autophagy suppresses the proliferation and tumorigenicity of human CRC cells $(17,18)$. The exact effects of RI on autophagy and the functional association of autophagy with RI remains largely unknown. The present study investigated the effect of RI on autophagy in CRC cells, and identified autophagy-associated proteins and pathways to assist in the identification of biological targets of human CRC.

\section{Materials and methods}

Reagents and antibodies. MK-2206 was purchased form Abmole Bioscience, Inc. (Houston, TX, USA). Bafilomycin A1 (BafiA1) was purchased from Sigma-Aldrich (Merck KGaA, Darmstadt, Germany). DMSO and DAPI were purchased from Beyotime Institute of Biotechnology (Haimen, China). Anti-RI (cat. no. ab245627), anti-autophagy-related protein (ATG)5 (cat. no. ab228668), anti-ATG7 (cat. no. ab223365), anti-ATG13 (cat. no. ab201467), anti-beclin-1 (BECN1; cat. no. ab62557), anti-LC3/II (cat. no. ab51520), anti- $\beta$-Actin (cat. no. ab119716) and goat anti-rabbit immunoglobulin G (IgG; Alexa Fluor ${ }^{\circledR}$ 488-conjugated; cat. no. ab150077) antibodies were purchased 
from Abcam (Cambridge, UK). Anti-Akt (cat. no. 9272), anti-phosphorylated (p)-Akt (cat. no. 9271), anti-mTOR (cat. no. 2972), anti-p-mTOR (cat. no. 2971), anti-ULK1 (cat. no. 6888), anti-p-ULK1 (cat. no. 5869), anti-AMPK (cat. no. 2532 S), anti-p-AMPK (cat. no. 2535), anti-P62/sequestosome 1 (SQSTM1; cat. no. 38749), goat anti-rabbit IgG horseradish peroxidase (HRP)-conjugated (cat. no. 7074) and goat anti-mouse IgG HRP-conjugated (cat. no. 7076) antibodies were purchased from Cell Signaling Technology, Inc. (Danvers, MA, USA).

Cell culture. The human CRC cell line HT29 was obtained from The Cell Bank of Type Culture Collection of Chinese Academy of Sciences (Shanghai, China) and was cultured in RPMI1640 Medium (Thermo Fisher Scientific, Inc., Waltham, MA, USA) supplemented with 10\% fetal bovine serum (Beijing Solarbio Science \& Technology Co., Ltd., Beijing, China) and $100 \mathrm{U} / \mathrm{ml}$ penicillin/streptomycin at $37^{\circ} \mathrm{C}$ in a humidified atmosphere containing $5 \% \mathrm{CO}_{2}$. Cells were treated with vehicle $(0.1 \%$ DMSO) or Akt inhibitor MK-2206 $(5 \mu \mathrm{M})$ at $37^{\circ} \mathrm{C}$ for $24 \mathrm{~h}$, and the levels of LC3-II were examined by western blotting. Cells were seeded at a density of $2 \times 10^{5}$ cells/well into six-well plates and were treated with DMSO $(0.1 \%)$ or bafilomycin A1 $(1 \mu \mathrm{M})$ at $37^{\circ} \mathrm{C}$ for $72 \mathrm{~h}$.

Overexpression of human RI. HT29 cells were seeded into six-well plates, and the pcDNA3.1-RI plasmid $(4 \mu \mathrm{g} / \mu \mathrm{l})$, maintained in the laboratory (12), was transfected using Lipofectamine ${ }^{\mathrm{TM}} 2000$ (Thermo Fisher Scientific, Inc.). Approximately $48 \mathrm{~h}$ after transfection, $800 \mu \mathrm{g} / \mathrm{ml}$ of geneticin (G418) (Sigma-Aldrich; Merck KGaA) was added to the medium and incubated for 2 weeks to select the positive clones that would be employed in generating a stable transfected cell line, HT29/RI, that overexpresses RI. Mock cells were transfected with an empty vector and used as a control (HT29/vector).

Short hairpin RNA (shRNA)-mediated knockdown assays. All of the plasmids required for shRNA-mediated knockdown assays were reconstructed and provided by Genepharm, Inc. (Sunnyvale, CA, USA). The pGPU6/GFP/Neo-shRNA vectors used to knock down RI, ATG13, BECN1, mTOR and ULK1 were shRI $(4 \mu \mathrm{g} / \mu \mathrm{l})$, shATG13 $(5 \mu \mathrm{g} / \mu \mathrm{l})$, shBECN1 $(4 \mu \mathrm{g} / \mu \mathrm{l})$, $\operatorname{shmTOR}(4 \mu \mathrm{g} / \mu \mathrm{l})$ and shULK1 $(4 \mu \mathrm{g} / \mu \mathrm{l})$, respectively. The transfection reagent Lipofectamine ${ }^{\circledR} 2000$ was used to transfer plasmid DNA into the HT29 cells. Non-targeting shRNA was used as negative control (shCTL; $4 \mu \mathrm{g} / \mu \mathrm{l})$. Cells were harvested $48 \mathrm{~h}$ following transfection.

Western blotting. The cells were harvested, and total cell lysates were prepared using a protein extraction kit (Nanjing KeyGen Biotech Co., Ltd., Nanjing, China) according to the manufacturer's protocol. Protein concentrations were quantified using a bicinchoninic acid kit (Beyotime Institute of Biotechnology). Equal amounts (20 $\mu \mathrm{g}$ per lane) of protein samples were separated using $8-12 \%$ SDS-PAGE and transferred onto nitrocellulose membranes. The membranes were blocked in TBS with $3 \%$ nonfat milk for $2 \mathrm{~h}$ at $37^{\circ} \mathrm{C}$, followed by incubation with primary antibodies at $4^{\circ} \mathrm{C}$ overnight. The primary antibodies used for western blotting were the following:
Anti-RI (1:2,000), anti-ATG5 (1:500), anti-ATG7 (1:1,000), anti-ATG13(1:1,000), anti-(BECN1) (1:600), anti-LC3/II $(1: 3,000)$, anti- $\beta$-Actin $(1: 1,000)$ anti-Akt $(1: 500)$, anti-p-Akt $(1: 1,000)$, anti-mTOR $(1: 1,000)$, anti-p-mTOR $(1: 1,000)$, anti-ULK1 (1:500), anti-p-ULK1 (1:500), anti-AMPK (1:500), anti-p-AMPK (1:500), anti-P62/SQSTM1 $(1: 1,000)$. The following secondary antibodies were incubated for $1 \mathrm{~h}$ at $37^{\circ} \mathrm{C}$ : Goat anti-rabbit HRP-linked IgG $(1: 2,000)$ or goat anti-mouse HRP-conjugated IgG $(1: 2,000)$. Chemiluminescent signals were detected using the Enhanced Chemiluminescent Plus kit (GE Healthcare, Chicago, IL, USA) and the signal intensity was measured by densitometric analysis using the Versa-Doc ${ }^{\text {TM }}$ Imaging system (version 4.0; Bio-Rad Laboratories, Inc., Hercules, CA, USA). $\beta$-actin was used as the internal control to normalize the samples.

Immunofluorescence staining. Cells were grown in six-well plates and fixed with 3.5\% formaldehyde in PBS for $10 \mathrm{~min}$ at room temperature, washed twice with PBS, permeabilized with $0.1 \%$ Triton X-100 for 10 min, and blocked with $0.5 \%$ bovine serum albumin (Beyotime Institute of Biotechnology) for $15 \mathrm{~min}$ at room temperature. Following two washes with PBS, the cells were incubated with an LC3-II primary-antibody (1:500) for $2 \mathrm{~h}$ at room temperature, followed by incubation with an Alexa Fluor ${ }^{\circledast}$ 488-conjugated secondary antibody (1:200) for $1 \mathrm{~h}$ at room temperature. Fluorescent signals were detected using a fluorescence microscope (Olympus Corporation, Tokyo, Japan; magnification, x400). Autophagy was quantified by counting the number of LC3-II dots per cell (a minimum of 50 cells per preparation in three independent experiments).

Colony-formation assay. For the clonogenic assay, cells were transfected with control shRNA (shCTL), RI shRNA (shRI), RI plasmid (RI) or empty vector (Vector) for $48 \mathrm{~h}$ respectively. Cells in different groups were subsequently seeded with appropriate dilutions into $6 \mathrm{~cm}$ dishes, followed by incubation at $37^{\circ} \mathrm{C}$ for 2 weeks. Colonies were fixed with $4 \%$ paraformaldehyde at $4^{\circ} \mathrm{C}$ for $30 \mathrm{~min}$, and subsequently stained with crystal violet $(0.5 \% \mathrm{w} / \mathrm{v})$ at room temperature for $5 \mathrm{~min}$. The dishes were imaged using a light microscope (Olympus Corporation; magnification, $\mathrm{x} 400$ ) and the numbers of distinct colonies ( $\geq 50$ cells per colony) were counted under the microscope (small, $<0.3 \mathrm{~mm}$; medium, 0.3-0.6 mm; large, $>0.6 \mathrm{~mm}$ ). The results were averaged for each treatment group.

Cell viability assay. The cell viability was determined by Cell Counting Kit-8 (CCK-8) assay (Beyotime Institute of Technology). Briefly, cells in each group were plated at a density of 3,000 cells/well in 96-well plates, and incubated at $37^{\circ} \mathrm{C}$ with $5 \% \mathrm{CO}_{2}$ for $12,24,48$ and $72 \mathrm{~h}$. CCK- 8 solution $(10 \mu \mathrm{l})$ was added to each well, the optical absorbance was determined at $450 \mathrm{~nm}$ following a $3 \mathrm{~h}$ incubation using BioTek $^{\mathrm{TM}}$ Epoch $^{\mathrm{TM}}$ (BioTek Instruments, Inc., Winooski, VT, USA).

Statistical analysis. The data were analyzed using SPSS software 16.0 (SPSS, Inc., Chicago, IL, USA). The results are expressed as the mean \pm standard error of the mean. Each experiment was repeated at least three times and analyzed 
A
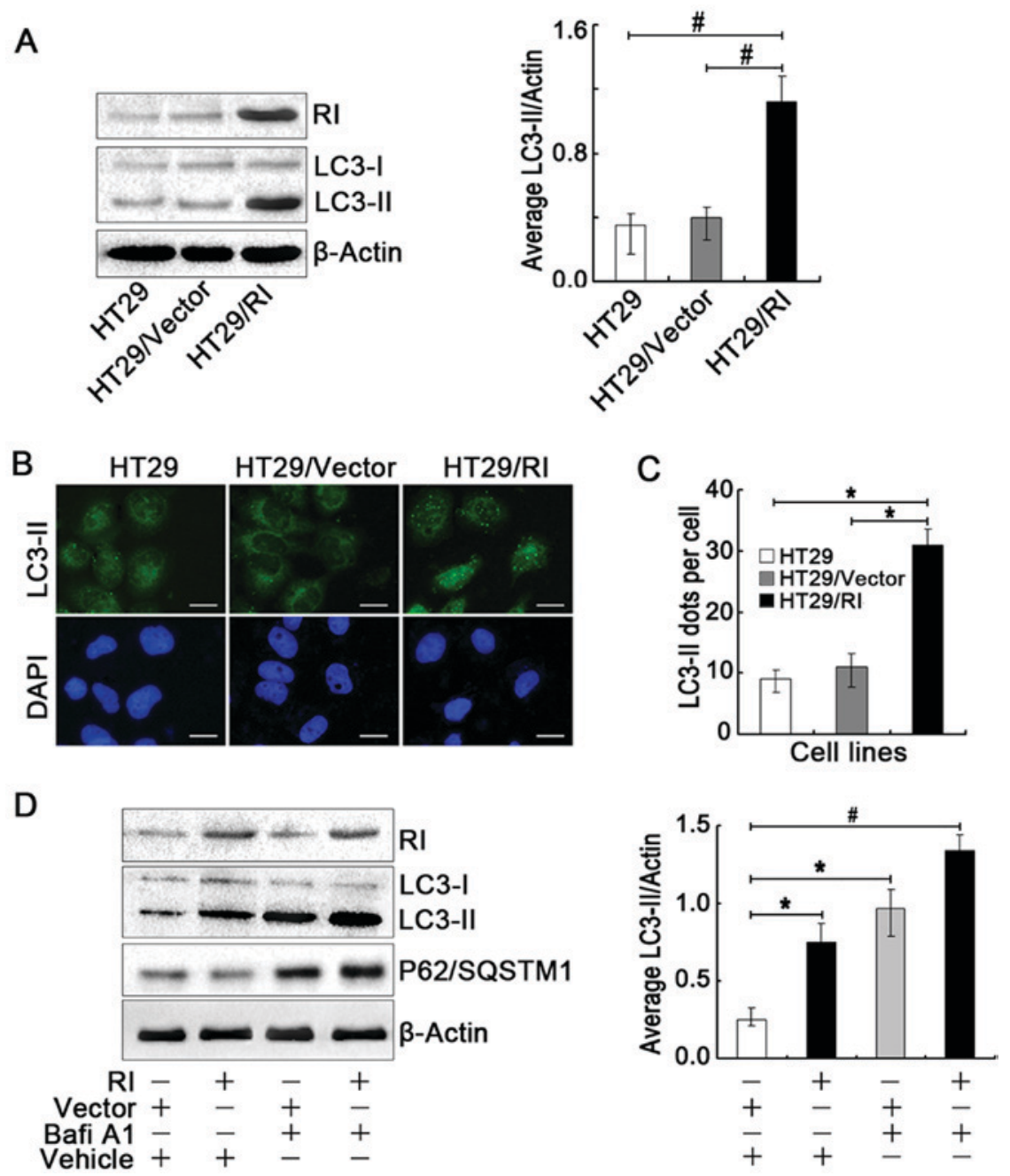

Figure 1. Overexpression of RI induces autophagy in HT29 cells. (A) RI, LC3-I and LC3-II (normalized to $\beta$-actin) were analyzed by western blotting. (B) Representative immunofluorescence images demonstrating the redistribution of autophagic marker LC3-II in HT29/RI cells. Images were acquired using a confocal microscope. Scale bar, $10 \mu \mathrm{m}$. (C) Average number of LC3-II dots/cell were counted in more than five fields (n 100 for each group). (D) LC3-II and P62/SQSTM1 protein levels were assessed by western blotting. HT-29/Vector or HT-29/RI cells were seeded at a density of 2x105 cells/well into six-well plates and were treated with DMSO $(0.1 \%)$ or bafilomycin $\mathrm{A} 1(1 \mu \mathrm{M})$ at $37^{\circ} \mathrm{C}$ for $72 \mathrm{~h}$. All quantitative data are presented as the mean \pm standard error of the mean of at least three independent experiments. ${ }^{*} \mathrm{P}<0.05$ and ${ }^{\text {"}} \mathrm{P}<0.01$ vs. respective control group. RI, ribonuclease inhibitor; LC3, light chain 3 ; Vector, control vector transfection; SQSTM1, sequestosome 1.

using a Students' t-test or one-way analysis of variance followed by Tukey's test for multiple comparisons. $\mathrm{P}<0.05$ was considered to indicate a statistically significant difference.

\section{Results}

Overexpression of RI induces autophagy in HT29 CRC cells. Previous studies have demonstrated that Akt inhibition strongly activates autophagy (19), and that overexpression of RI suppresses the PI3K/Akt pathway in human CRC cells (12). To study a possible regulatory effect of RI on autophagy in HT29 cells, these cells were transfected with pcDNA3.1-RI recombinant vectors, and successfully transfected cells were selected for further analyses. Overexpression of RI markedly increased LC3-II levels in the HT29 cells (Fig. 1A), suggesting that increased RI expression induces autophagy. In addition, fluorescence microscopy was used to verify the generation of autophagosomes. An increase in the number of LC3-II puncta was observed in the HT29/RI cells (Fig. 1B), further substantiating the previous observations. As visible in Fig. 1B, the majority of LC3-II puncta were concentrated in the nucleus. The number of LC3-II puncta/cell significantly increased by $>3$-fold compared with the HT29 and HT29/Vector cells controls (Fig. 1C). LC3-II levels were also elevated during autophagosome-lysosome fusion or autophagic vesicle degradation. To verify that this LC3-II elevation was induced by the upregulation of RI, the autophagic flux in the HT29/RI cells was analyzed in the absence (Vehicle) or presence of BafiA1, an inhibitor of autophagosome-lysosome fusion and LC3-II degradation. As indicated in Fig. 1D, BafiA1 increased the levels of LC3-II and of the autophagy-specific substrate P62/SQSTM1 compared with the control and empty vector groups, suggesting that LC3-II accumulation in HT29/RI cells was attributable to the promotion of autophagy but not to autophagic degradation. Taken together, these results confirmed that the overexpression of RI triggered autophagy in human CRC cells.

RI regulates HT29 cell survival. A previous study demonstrated that the upregulation of RI affects the morphology and proliferation of bladder cancer cells (20). To investigate 

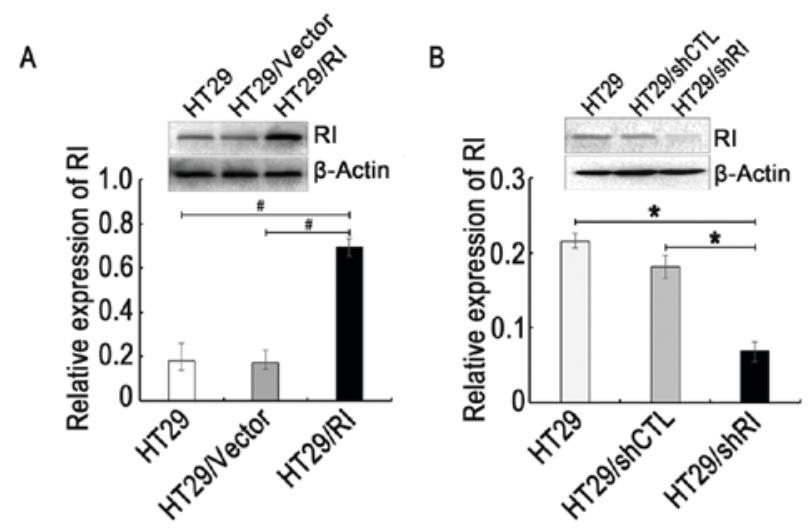

C

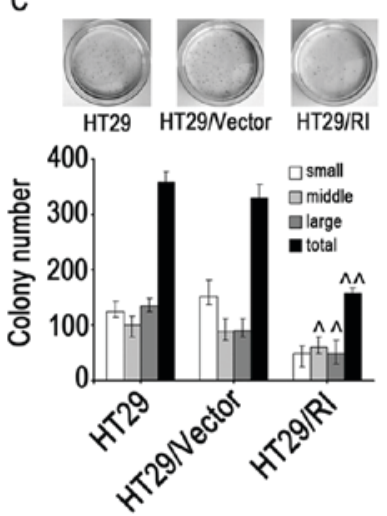

D
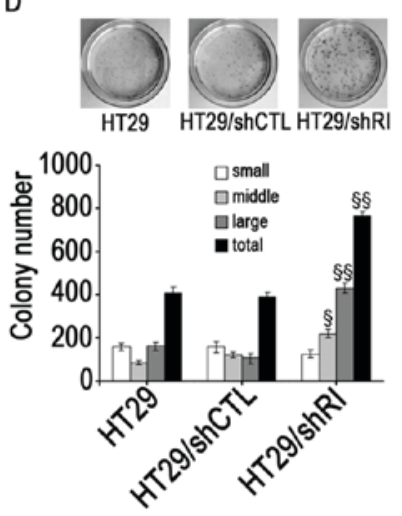

$\mathrm{E}$

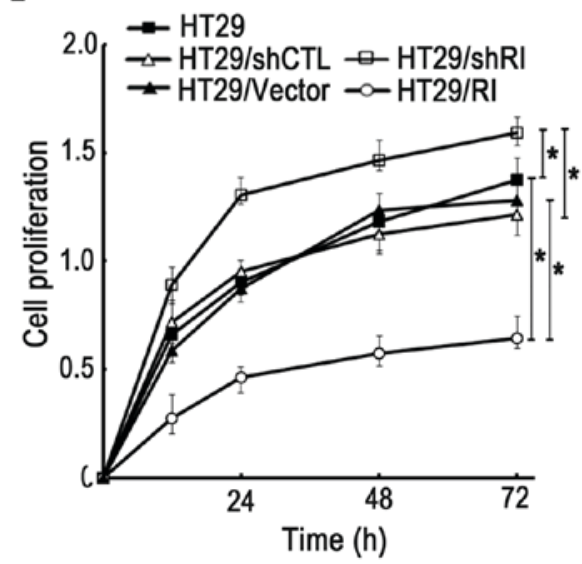

Figure 2. RI regulates HT29 cell survival. Western blotting was used to assess the effectiveness of transfections of (A) a plasmid overexpressing RI and (B) shRI downregulating RI in HT29 cells. The effects of (C) RI overexpression and (D) RI knockdown on colony formation were assessed. Cell colonies were photographed and counted under a microscope, and divided according to the size (small, $<0.3 \mathrm{~mm}$; medium, $0.3-0.6 \mathrm{~mm}$; large, $>0.6 \mathrm{~mm}$ ). A colony was defined as a cluster of $\geq 50$ cells. (E) The effects of RI knockdown and $\mathrm{RI}$ overexpression on cell viability were determined using the Cell Counting Kit- 8 assay at defined time points. All quantitative data are presented as the mean \pm standard error of the mean of at least three independent experiments. ${ }^{*} \mathrm{P}<0.05$ and ${ }^{\#} \mathrm{P}<0.01$ vs. respective control group. ${ }^{\wedge} \mathrm{P}<0.05$ and ${ }^{\wedge} \mathrm{P}<0.01$ vs. HT29/Vector. ${ }^{\circledR} \mathrm{P}<0.05$ and ${ }^{\S \S} \mathrm{P}<0.01$ vs. HT29/shCTL. RI, ribonuclease inhibitor; shRI, short hairpin RNA targeting RI; Vector, control vector transfection; shCTL, non-targeting short hairpin RNA used as a control.

the effects of RI on HT29 cell survival, RI expression was upregulated by introducing an exogenous $R I$ gene using the overexpression vector pcDNA3.1/RI, or silenced using a specific shRNA (shRI)-mediated knockdown. As presented in Fig. 2A and B, RI expression levels significantly increased or decreased in the HT29/RI or HT29/shRI cells, respectively, compared with the controls, suggesting that the transfection and RI expression manipulation were successful.

A colony formation assay was conducted to elucidate the association between RI expression and HT29 cell metastasis. The overexpression of RI significantly inhibited CRC cell colony formation, whereas knocking down RI in HT29 cells increased it, indicating the inhibitory effect of RI on HT29 cell tumorigenic ability (Fig. 2C and D). Cell viability was subsequently assessed using the CCK-8 assay. The results further provided the evidence that RI expression is negatively associated with viability in CRC cells (Fig. 2E). These results therefore supported the observations made with the colony formation assay, demonstrating that RI overexpression may negatively affect the viability and tumorigenic abilities of CRC cells.

Autophagy-associated proteins BECN1 and ATG13 are essential for autophagy in response to RI overexpression in HT29 cells. To determine whether autophagy induced by RI overexpression contributes to the regulation of specific proteins of the ATG family, the expression levels of ATG5, ATG7, ATG13 and BECN1 were assessed by western blotting. Fig. 3A indicates that overexpression of RI significantly increased the protein levels of BECN1 and ATG13, but not ATG5 and ATG7. To further validate these observations, specific shRNA sequences of ATG13 (shATG13) and BECN1 (shBECN1) were transfected into HT29/RI cells. The results demonstrated that LC3-II levels significantly decreased due to the knockdown of BECN1 and ATG13 in the HT29/RI cells (Fig. 3B and C). Furthermore, the formation of LC3-II autophagic vacuoles were observed under fluorescence microscopy. As exhibited in Fig. 3D, the knockdown of ATG13 or BECN1 significantly attenuated the accumulation of LC3-II in RI-overexpressing cells. Moreover, the number of LC3-II dots/cell significantly decreased following silencing of ATG13 or BECN1 in HT29/RI cells. Taken together, these results indicated that ATG13 and BECN1 may have been responsible for autophagy induced by $\mathrm{RI}$ overexpression in human CRC cells.

Akt/mTOR/ULK1 pathway is involved in the activation of autophagy in CRC cells. To evaluate which pathways may have mediated the effect of RI on autophagy and cell viability, signal transduction molecules, including Akt, mTOR, ULK1 and AMPK, were examined in CRC cells. A previous study demonstrated that the function of RI is associated with the PI3K/Akt pathway (12). Consistent with these reports, the present study revealed that Akt and mTOR were significantly inactivated in HT29/RI cells, whereas ULK1 was activated by phosphorylation, but there was no effect on the expression levels of phosphorylated AMPK (p-AMPK), total Akt, total mTOR, total ULK1 and total AMPK (Fig. 4A). These results suggested that RI overexpression may have induced autophagy by inhibiting the Akt/mTOR/ULK1 pathway, but not via the activation of AMPK. To further validate whether Akt, mTOR and its downstream target ULK1 were involved in RI induced autophagy, the effects of the Akt inhibitor, MK-2206 (21), were evaluated by assessing its effects on LC3-II levels. Fig. 4B demonstrates that the inhibition of activated Akt (p-Akt) resulted in an increase in the amount of LC3-II. In 
A
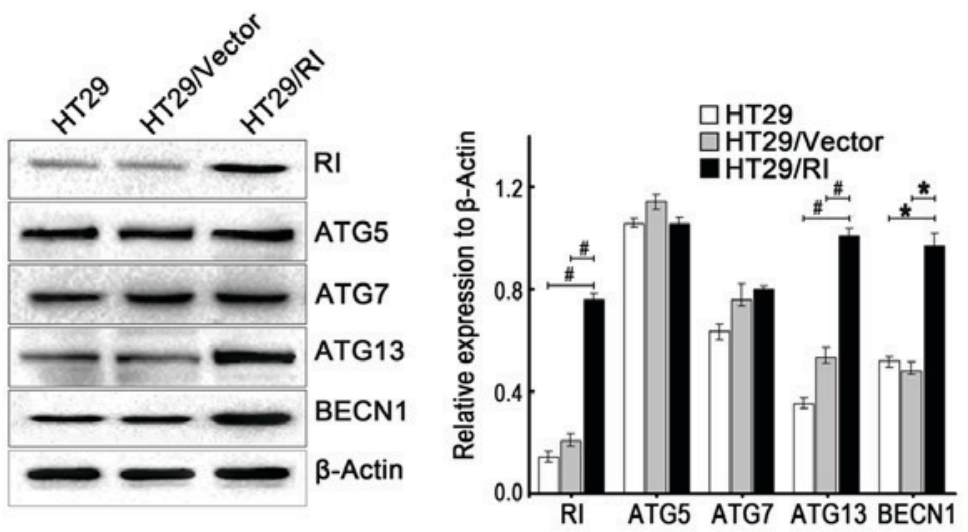

B
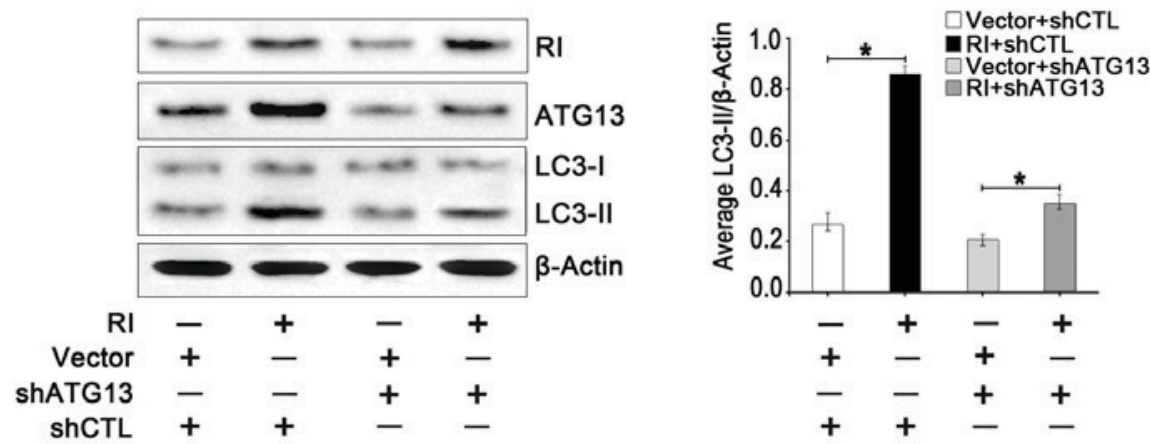

C
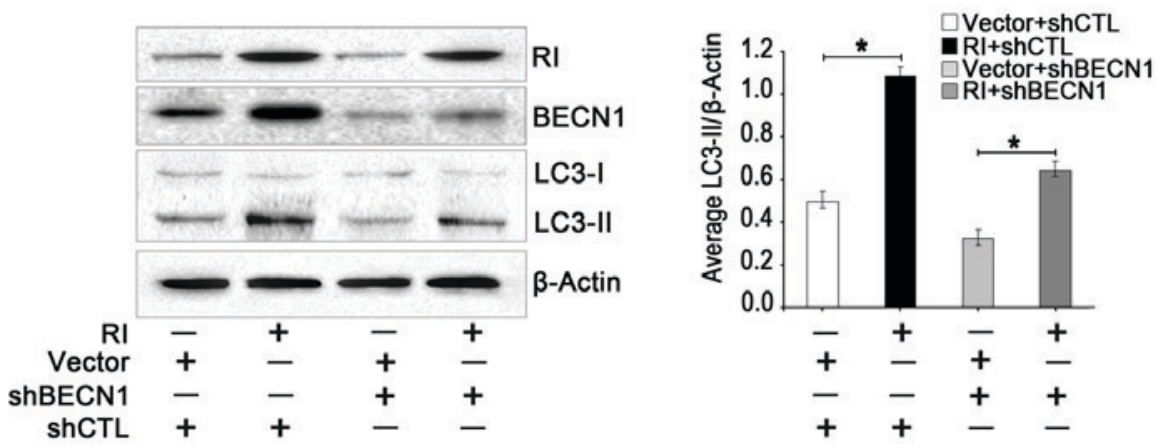

D
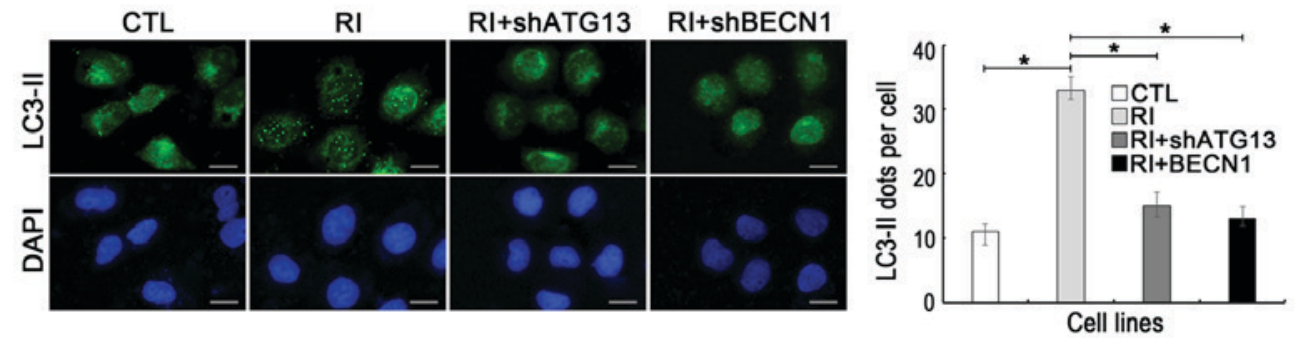

Figure 3. ATG13 and BECN1 are required for autophagy in response to RI overexpression in HT29 cells. (A) The protein levels of ATG5, ATG7, ATG13 and BECN1 (normalized to $\beta$-actin) were analyzed by western blotting. Representative western blot and densitometric analyses normalized to $\beta$-actin demonstrating the effects of (B) shATG13 and (C) shBECN1 on LC3-II levels following RI overexpression. (D) Effect of shATG13 and shBECN1 on LC3-II accumulation induced by RI overexpression compared to the CTL. The average number of LC3-II dots/cell were counted in more than five fields with $\geq 100$ cells in each group. All quantitative data are presented as the mean \pm standard error of the mean of at least three independent experiments. ${ }^{*} \mathrm{P}<0.05$ and ${ }^{\#} \mathrm{P}<0.01$ vs. respective control group. RI, ribonuclease inhibitor; LC3, light chain 3; ATG, autophagy-related protein; BECN1, beclin 1; shATG13, short hairpin RNA targeting ATG13; shBECN1, short hairpin RNA targeting BECN1; Vector, control vector transfection; shCTL, non-targeting short hairpin RNA used as a control; CTL, control.

addition, the downregulation of mTOR (shmTOR) increased ULK1 phosphorylation and significantly promoted LC3-II accumulation (Fig. 4C), whereas silencing of ULK1 markedly reduced LC3-II levels, indicating that mTOR and ULK1, as the downstream elements of Akt signaling, may also be involved in the process of RI-induced autophagy 
A
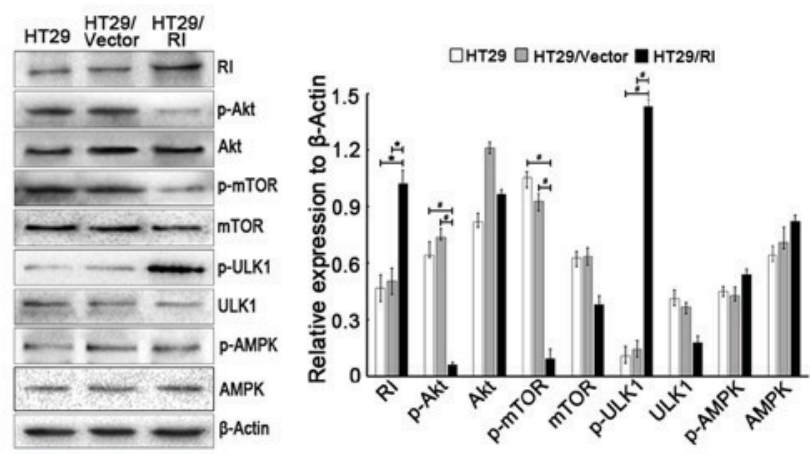

B
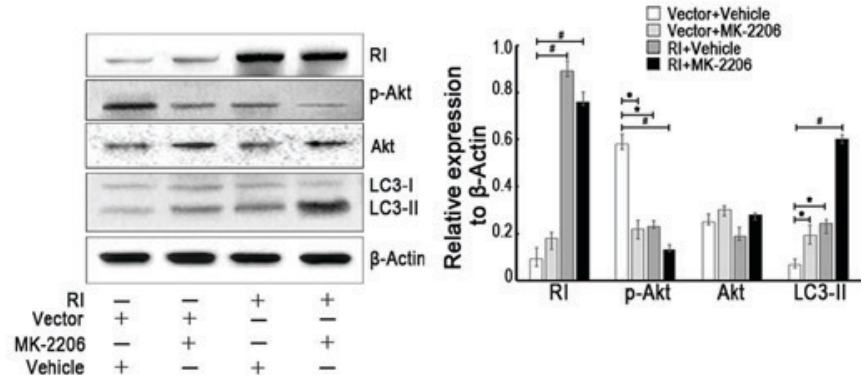

D

C

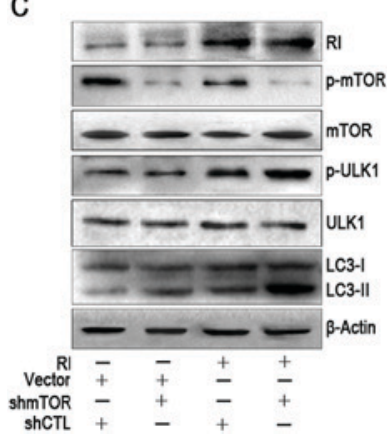

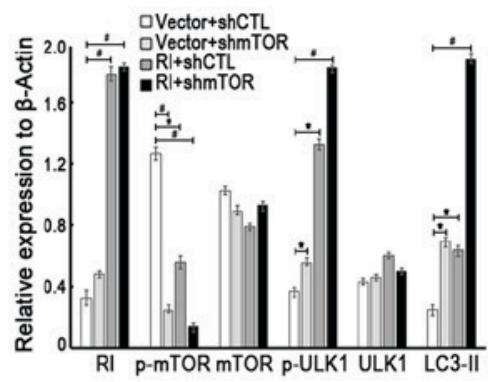

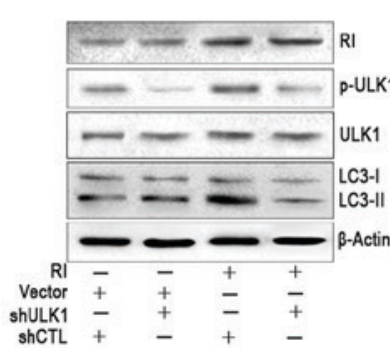

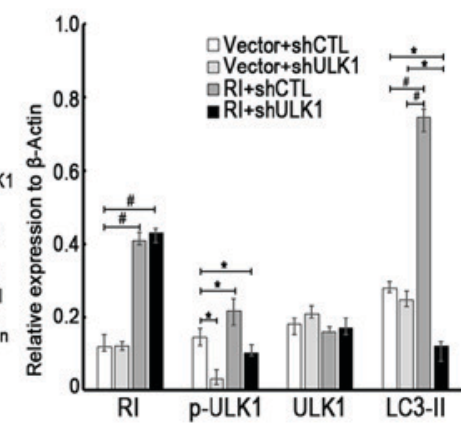

Figure 4. Akt/mTOR/ULK1 signaling pathway is responsible for RI-induced autophagy in colorectal cancer cells. (A) The protein levels of RI, p-Akt, Akt, p-mTOR, mTOR, p-ULK1, ULK1, p-AMPK and AMPK (normalized to $\beta$-actin) were analyzed by western blotting. (B) HT-29/Vector or HT-29/RI cells were treated with vehicle (0.1\% DMSO) or Akt inhibitor MK-2206 $(5 \mu \mathrm{M})$ at $37^{\circ} \mathrm{C}$ for $24 \mathrm{~h}$, and the levels of LC3-II were examined by western blotting. Representative figures demonstrating the effect of inhibition of p-Akt on LC3-II accumulation compared with the control groups. Representative western blotting images indicating the effect of (C) shmTOR and (D) shULK1 on LC3-II levels. All quantitative data are presented as the mean \pm standard error of the mean of at least three independent experiments. " $\mathrm{P}<0.05$ and ${ }^{\#} \mathrm{P}<0.01$. RI, ribonuclease inhibitor; Akt, protein kinase B; mTOR, mechanistic target of rapamycin; ULK1, Unc-51 like autophagy activating kinase; AMPK, adenosine monophosphate-activated protein kinase; p-, phosphorylated; LC3, light chain 3; shmTOR, short hairpin RNA targeting mTOR; shULK1, short hairpin RNA targeting ULK1.

in CRC cells (Fig. 4D). Based on these results, activation of the Akt/mTOR/ULK1 signaling pathway may have been responsible for RI-induced autophagy in CRC cells.

\section{Discussion}

$\mathrm{RI}$ is a cytoplasmic acidic protein that is involved in multiple biological processes, including inhibition of RNase A and ANG activity. Previous studies have demonstrated that RI regulates stress-induced subcellular localization of angiogenin to control growth and survival of HeLa cells (22), and that upregulation of RI inhibits the growth and metastasis in melanoma, breast cancer and bladder cancer cells $(8,20,23)$. However, the effect of RI on the growth of colorectal cancer cells and its underlying mechanisms, were not fully understood. In the present study, RI protein expression level was significantly decreased in HT29 cells compared with other cell lines, including HCT116, CW-2 and LoVo as assessed by western blotting (data not presented); therefore, HT29 cells were cultured and transfected, and served as the primary model to study the potential effects of RI on autophagy in CRC cells. Furthermore, the present study demonstrated that RI overexpression induces autophagy in human CRC cells, possibly via the Akt/mTOR/ULK1 signaling pathway.

Autophagy is a critical event which maintains tissue homeostasis under basal conditions (24). Although the precise role of autophagy in cancer cells remains unclear, previous studies have demonstrated that autophagy appears to be a double-edged sword that may be beneficial or detrimental to cancer development (25). Earlier investigations have reported that RI serves a noteworthy role in ANG-induced angiogenesis $(26,27)$, depleting nutrients that may be utilized by cells. Alternatively, autophagy provides energy by degrading intracellular macromolecules, proteins and organelles, which may be beneficial to the growth of tumor cells in a low vascular environment $(25,28)$. Therefore, defining the context-specific role of autophagy in CRC cells and the association between RI and autophagy may guide autophagy-based therapeutic interventions. In the present study, overexpression of RI was observed to induce autophagy in human CRC cells.

Specific autophagy-associated genes are involved in this process. Studies have indicated that more than 40 ATG proteins are involved in autophagosome formation (29). Previous studies have also reported that autophagy may be induced through ATG5, BECN1 or ATG7-dependent or -independent signaling pathways (30), whereas ATG13, one of the components of the ULK1 kinase complex, is required for the initiation of autophagosome formation (31). The present study assessed the expression of ATG5, ATG7, BECN1 and ATG13 in response to RI overexpression, and revealed that only BECN1 and ATG13 may be required for CRC cell autophagy, suggesting that the ULK1 complex may be involved in this process.

Autophagy involves three stages, including initiation, elongation and maturation (32). Various signaling pathways have been implicated in the regulation of 
autophagy (33-35). mTOR inhibits autophagy by forming a protein complex associated with ULK1 (also termed the 'preinitiation' complex), which is the central molecule that is involved in autophagy regulation $(36,37)$. This complex may be negatively and positively regulated by a variety of intracellular signals. The PI3K pathway is also known to be involved in autophagy, as the inhibition of PI3K/Akt and mTOR were demonstrated to upregulate autophagy in breast cancer cells $(38,39)$. AMPK, which serves a role in the regulation of cellular lipid and protein metabolism, also induces autophagy by activating ULK1 or by inactivating mTOR (36). In addition, mTOR may suppress ULK1 under certain conditions $(40,41)$. As previously reported, the inhibition of Akt may induce autophagy, and may therefore exert anti-tumor activity $(12,42)$. Furthermore, the present study revealed that autophagy induced by RI is dependent on the mTOR/ULK1 pathway, but not on the activation of AMPK.

Taken together, the results of the present study indicated that the upregulation of RI promoted the expression of autophagy-associated genes, including BECN1 and ATG13, and prevented phosphorylation of Akt and mTOR, which in turn may have alleviated the inhibition of ULK1, ultimately leading to autophagy in CRC cells. Therefore, the upregulation of RI may constitute a novel strategy for the treatment of human CRC.

\section{Acknowledgements}

Not applicable.

\section{Funding}

This study was supported by a grant from the National Science Foundation of China (grant no. 31400687).

\section{Availability of data and materials}

The datasets used and/or analyzed during the current study are available from the corresponding author on reasonable request.

\section{Authors' contributions}

YiT and FR performed the experiments and wrote the manuscript. XC, YK, YX and YuT performed the experiments and performed statistical analysis. JF designed the study and critically reviewed the manuscript. All authors read and approved the final version of manuscript.

\section{Ethics approval and consent to participate}

Not applicable.

\section{Patient consent for publication}

Not applicable.

\section{Competing interests}

The authors declare that they have no competing interests.

\section{References}

1. Siegel RL, Miller KD and Jemal A: Cancer statistics, 2016. CA Cancer J Clin 66: 7-30, 2016.

2. Chen W, Zheng R, Baade PD, Zhang S, Zeng H, Bray F, Jemal A, $\mathrm{Yu}$ XQ and He J: Cancer statistics in China, 2015. CA Cancer J Clin 66: 115-132, 2016.

3. Ciombor $\mathrm{KK}, \mathrm{Wu} \mathrm{C}$ and Goldberg RM: Recent therapeutic advances in the treatment of colorectal cancer. Annu Rev Med 66: 83-95, 2015.

4. Kobe B and Deisenhofer J: Crystal structure of porcine ribonuclease inhibitor, a protein with leucine-rich repeats. Nature 366 : 751-756, 1993.

5. McEwan PA, Scott PG, Bishop PN and Bella J: Structural correlations in the family of small leucine-rich repeat proteins and proteoglycans. J Struct Biol 155: 294-305, 2006.

6. Datta D, Samanta A, Dasgupta S and Pathak T: 3'-Oxo-, amino-, thio- and sulfone-acetic acid modified thymidines: Effect of increased acidity on ribonuclease A inhibition. Bioorg Med Chem 21: 4634-4645, 2013.

7. Dickson KA, Haigis MC and Raines RT: Ribonuclease inhibitor: Structure and function. Prog Nucleic Acid Res Mol Biol 80: 349-374, 2005

8. Pan X, Xiong D, Yao X, Xin Y,Zhang L and Chen J: Up-regulating ribonuclease inhibitor inhibited epithelial-to-mesenchymal transition and metastasis in murine melanoma cells. Int J Biochem Cell Biol 44: 998-1008, 2012.

9. Yao X, Li D, Xiong DM, Li L, Jiang R and Chen JX: A novel role of ribonuclease inhibitor in regulation of epithelial-to-mesenchymal transition and ILK signaling pathway in bladder cancer cells. Cell Tissue Res 353: 409-423, 2013.

10. Chen JX, Gao Y, Liu JW, Tian YX, Zhao J and Cui XY: Antitumor effects of human ribonuclease inhibitor gene transfected on B16 melanoma cells. Int J Biochem Cell Biol 37: 1219-1231, 2005.

11. Tian YX, Wang DM and Cui XY: Analysis of gene expression of ribonuclease inhibitor in human breast cancer tissue. Ai Zheng 23: 269-272, 2004 (In Chinese).

12. Tang Y,Liu P, Tian Y,Xu Y,Ren F, Cui X andFan J: Overexpression of ribonuclease inhibitor defines good prognosis and suppresses proliferation and metastasis in human colorectal cancer cells via PI3K/AKT pathway. Clin Transl Oncol 17: 306-313, 2015.

13. Degtyarev M, De Mazière A, Orr C, Lin J, Lee BB, Tien JY, Prior WW, van Dijk S, Wu H, Gray DC, et al: Akt inhibition promotes autophagy and sensitizes PTEN-null tumors to lysosomotropic agents. J Cell Biol 183: 101-116, 2008.

14. Kabeya Y, Mizushima N, Ueno T, Yamamoto A, Kirisako T, Noda T, Kominami E, Ohsumi Y and Yoshimori T: LC3, a mammalian homologue of yeast Apg8p, is localized in autophagosome membranes after processing. EMBO J 19: 5720-5728, 2000.

15. Mizushima N, Levine B, Cuervo AM and Klionsky DJ: Autophagy fights disease through cellular self-digestion. Nature 451: 1069-1075, 2008.

16. White E and DiPaola RS: The double-edged sword of autophagy modulation in cancer. Clin Cancer Res 15: 5308-5316, 2009.

17. Ellington AA, Berhow M and Singletary KW: Induction of macroautophagy in human colon cancer cells by soybean B-group triterpenoid saponins. Carcinogenesis 26: 159-167, 2005.

18. Liang C, Feng P, Ku B, Dotan I, Canaani D, Oh BH and Jung JU: Autophagic and tumour suppressor activity of a novel Beclin1-binding protein UVRAG. Nat Cell Biol 8: 688-699, 2006.

19. Cheng Y, Ren X, Zhang Y, Patel R, Sharma A, Wu H, Robertson GP, Yan L, Rubin E and Yang JM: eEF-2 kinase dictates cross-talk between autophagy and apoptosis induced by Akt Inhibition, thereby modulating cytotoxicity of novel Akt inhibitor MK-2206. Cancer Res 71: 2654-2663, 2011.

20. Zhuang X, Lv M, Zhong Z, Zhang L, Jiang R and Chen J: Interplay between intergrin-linked kinase and ribonuclease inhibitor affects growth and metastasis of bladder cancer through signaling ILK pathways. J Exp Clin Cancer Res 35: 130, 2016.

21. Ji S, Lin W, Wang L, Ni Z, Jin F, Zha X and Fei G: Combined targeting of mTOR and Akt using rapamycin and MK-2206 in the treatment of tuberous sclerosis complex. J Cancer 8: 555-562, 2017.

22. Pizzo E, Sarcinelli C, Sheng J, Fusco S, Formiggini F, Netti P, Yu W, D'Alessio G and Hu GF: Ribonuclease/angiogenin inhibitor 1 regulates stress-induced subcellular localization of angiogenin to control growth and survival. J Cell Sci 126: 4308-4319, 2013. 
23. Zhou JH, Tang XY, Zhao R, Wang H and Xia J: Effects of ribonuclease inhibitor on apoptosis and invasion of human breast cancer MDA-MB-231 cells. Xi Bao Yu Fen Zi Mian Yi Xue Za Zhi 28: 260-264, 2012 (In Chinese).

24. Han K, Kim J and Choi M: Autophagy mediates phase transitions from cell death to life. Heliyon 1: e00027, 2015.

25. Carroll RG and Martin SJ: Autophagy in multiple myeloma: What makes you stronger can also kill you. Cancer Cell 23: 425-426, 2013.

26. Dickson KA, Kang DK, Kwon YS, Kim JC, Leland PA, Kim BM, Chang SI and Raines RT: Ribonuclease inhibitor regulates neovascularization by human angiogenin. Biochemistry 48 : 3804-3806, 2009.

27. Peng Y, Li L, Huang M, Duan C, Zhang L and Chen J: Angiogenin interacts with ribonuclease inhibitor regulating $\mathrm{PI} 3 \mathrm{~K} / \mathrm{AKT} / \mathrm{mTOR}$ signaling pathway in bladder cancer cells. Cell Signal 26: 2782-2792, 2014.

28. Liu JL, Chang KC, Lo CC, Chu PY and Liu CH: Expression of autophagy-related protein Beclin-1 in malignant canine mammary tumors. BMC Vet Res 9: 75, 2013.

29. Young PG, Passalacqua MJ, Chappell K, Llinas RJ and Bartel B: A facile forward-genetic screen for Arabidopsis autophagy mutants reveals twenty-one loss-of-function mutations disrupting six ATG genes. Autophagy: 1-19, 2019.

30. Nishida Y, Arakawa S, Fujitani K, Yamaguchi H, Mizuta T, Kanaseki T, Komatsu M, Otsu K, Tsujimoto Y and Shimizu S: Discovery of Atg5/Atg7-independent alternative macroautophagy. Nature 461: 654-658, 2009.

31. Qi S, Kim DJ, Stjepanovic G and Hurley JH: Structure of the human Atg13-Atg101 HORMA heterodimer: An interaction hub within the ULK1 complex. Structure 23: 1848-1857, 2015.

32. Levine B: Cell biology: Autophagy and cancer. Nature 446 745-747, 2007

33. Liu M, Zhao G, Zhang D, An W, Lai H, Li X, Cao S and Lin X: Active fraction of clove induces apoptosis via $\mathrm{PI}$ K/Akt/mTOR-mediated autophagy in human colorectal cancer HCT-116 cells. Int J Oncol 53: 1363-1373, 2018.
34. Yang L, Liu Y, Wang M, Qian Y, Dai X, Zhu Y, Chen J, Guo S and Hisamitsu T: Celastrus orbiculatus extract triggers apoptosis and autophagy via PI3K/Akt/mTOR inhibition in human colorectal cancer cells. Oncol Lett 12: 3771-3778, 2016.

35. Fan XJ, Wang Y, Wang L and Zhu M: Salidroside induces apoptosis and autophagy in human colorectal cancer cells through inhibition of PI3K/Akt/mTOR pathway. Oncol Rep 36: 3559-3567, 2016.

36. Kim J, Kundu M, Viollet B and Guan KL: AMPK and mTOR regulate autophagy through direct phosphorylation of Ulk1. Nat Cell Biol 13: 132-141, 2011.

37. Egan DF, Shackelford DB, Mihaylova MM, Gelino S, Kohnz RA, Mair W, Vasquez DS, Joshi A, Gwinn DM, Taylor R, et al: Phosphorylation of ULK1 (hATG1) by AMP-activated protein kinase connects energy sensing to mitophagy. Science 331: 456-461, 2011

38. Lin Y, Kuang W, Wu B, Xie C, Liu C and Tu Z: IL-12 induces autophagy in human breast cancer cells through AMPK and the PI3K/Akt pathway. Mol Med Rep 16: 4113-4118, 2017

39. Zhou X, Yue GG, Chan AM, Tsui SK, Fung KP, Sun H, Pu J and Lau CB: Eriocalyxin B, a novel autophagy inducer, exerts anti-tumor activity through the suppression of $\mathrm{Akt} / \mathrm{mTOR} / \mathrm{p} 70 \mathrm{~S} 6 \mathrm{~K}$ signaling pathway in breast cancer. Biochem Pharmacol 142: $58-70,2017$

40. Mizushima N and Komatsu M: Autophagy: Renovation of cells and tissues. Cell 147: 728-741, 2011.

41. Green DR and Levine B: To be or not to be? How selective autophagy and cell death govern cell fate. Cell 157: 65-75, 2014.

42. Fujiwara K, Iwado E, Mills GB, Sawaya R, Kondo S and Kondo Y: Akt inhibitor shows anticancer and radiosensitizing effects in malignant glioma cells by inducing autophagy. Int J Oncol 31: 753-760, 2007.

(i) $($ ) This work is licensed under a Creative Common Attribution-NonCommercial-NoDerivatives 4.0 International (CC BY-NC-ND 4.0) License. 